\title{
Numerical study on the influence of entrapped air bubbles on the time- dependent pore pressure distribution in soils due to external changes in water level
}

\author{
Georg M. Ausweger ${ }^{1, a}$ and Helmut F. Schweiger ${ }^{1}$ \\ ${ }^{1}$ Graz University of Technology, Institute of Soil Mechanics and Foundation Engineering, Computational Geotechnics Group, 8010 Graz, \\ Austria
}

\begin{abstract}
In practical geotechnical engineering soils below the groundwater table are usually regarded as a two-phase medium, consisting of solids and water. The pore water is assumed to be incompressible. However, under certain conditions soils below the groundwater table may exhibit a liquid phase consisting of water and air. The air occurs in form of entrapped air bubbles and dissolved air. Such conditions are named quasi-saturated and the assumption of incompressibility is no longer justified. In addition the entrapped air bubbles influence the hydraulic conductivity of soils. These effects are usually neglected in standard problems of geotechnical engineering. However, sometimes it is required to include the pore fluid compressibility when modelling the hydraulic behaviour of soils in order to be able to explain certain phenomena observed in the field. This is for example true for fast fluctuating water levels in reservoirs. In order to study these phenomena, numerical investigations on the influence of the pore fluid compressibility on the pore water pressure changes in a soil layer beneath a reservoir with fast fluctuating water levels were performed. Preliminary results of this study are presented and it could be shown that numerical analysis and field data are in good agreement.
\end{abstract}

\section{Introduction}

In classical soil mechanics, soils below the ground water table are considered as a two-phase medium. All pores are filled with water and the pore water is assumed to be incompressible, at least for practical purposes. However, if a small amount of air is present in the pore water, the compressibility of the pore fluid and the hydraulic conductivity of the soil are influenced significantly. This air phase is discontinuous and occurs as small entrapped air bubbles and the air pressure in the air bubbles is no longer equal to the atmospheric pressure.

As soils below the ground water level with entrapped air bubbles are not fully saturated but the term unsaturated soils is in general used for zones in the subsoil with a continuous air phase and a suction higher than the air entry value, a new term "quasi-saturated soils" was introduced to describe soils with entrapped air bubbles in the pore water [1]

The term quasi-saturated soil is used for soils with positive pore water pressure and entrapped air bubbles as well as soils with a negative pore water pressure (suction) smaller than the air entry value. This contribution considers only states with a positive pore water pressure.

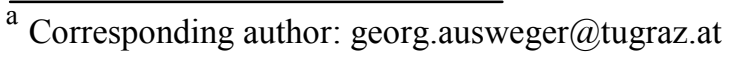

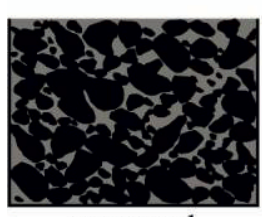

saturated

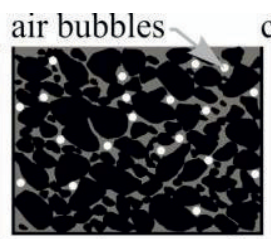

quasi-saturated

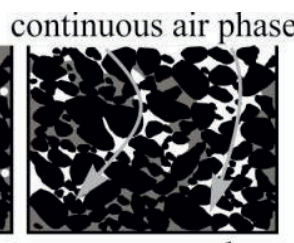

unsaturated
Figure 1. Comparison saturated - quasi-saturated - unsaturated soil. Adapted according to [2].

Observations in the field $[3,1]$ and laboratory tests [1, 4] show that soils below the ground water table are often in a quasi-saturated state. This has an influence on the behaviour of the pore fluid. The air bubbles in the soil can occur due to different reasons, for example fluctuating water tables [4]. Such fluctuating water tables are possible, amongst others, in storage basins of pumped storage power plants and in ship canals.

In this contribution the effects of air bubbles in the pore water are included in modelling the hydraulic behaviour of the soil as a two-phase medium. The aim is to explain, in a qualitative manner, the results of pore pressure measurements near a storage basin of a pumped storage power plant employing the finite element method. The focus is exclusively on matching the measured pore water pressures. In the first part of the paper the basics for the modelling of quasi-saturated soils are summarized for continuity. The second part presents the pore pressure 
measurement data and the comparison with results from finite element analyses.

\section{Modelling of quasi-saturated soils}

Soils in a quasi-saturated state consist of incompressible solids and a compressible pore fluid. The pore fluid is compressible due to entrapped air bubbles in the pore water. As the air bubbles reduce the hydraulic conductivity of soils, it is necessary to define a relative hydraulic conductivity $k_{\text {rel }}$, depending on the air content in the pore fluid. Furthermore, a water retention curve (WRC) has to be defined for quasi saturated soils.

The flow of pore fluid in a porous deformable material [5] can be described by

$$
\begin{gathered}
S \frac{\partial \varepsilon_{v o l}}{\partial t}-n \cdot\left(\frac{S}{K_{w}}-\frac{\partial S}{\partial p_{w}}\right) \cdot \frac{\partial p_{w}}{\partial t}+\cdots \\
\cdots \nabla^{T}\left[\frac{k_{r e l}}{\rho_{w} \cdot g} \mathbf{k}^{s a t} \cdot\left(\nabla p_{w}+\rho_{w} \cdot \mathbf{g}\right)\right]=0
\end{gathered}
$$

where $S$ is the degree of saturation, $\varepsilon_{v o l}$ are the volumetric strains, $K_{w}$ is the bulk modulus of water and $p_{w}$ is the pore water pressure. $k_{r e l}$ and $\mathbf{k}^{\text {sat }}$ represent the relative and saturated hydraulic conductivity respectively and $\rho_{w}$ is the density of water.

In the following the influence of entrapped air bubbles on the relative hydraulic conductivity and the WRC, i.e. the relationship between degree of saturation $S$ and pore water pressure $p_{w}$, is discussed.

\subsection{Hydraulic conductivity of quasi-saturated soils}

Laboratory tests on different soil types $[1,4,6,7]$ show a decrease in the hydraulic conductivity with increasing entrapped air content. Usually this decrease in hydraulic conductivity is described by a power law. An example for such a power law is the one according to Faybishenko [1].

$$
\mathbf{k}^{q s}=\mathbf{k}_{\mathbf{0}}+\left(\mathbf{k}^{s a t}-\mathbf{k}_{\mathbf{0}}\right) \cdot\left(1-\frac{\omega}{\omega_{\max }}\right)^{n}
$$

The quasi-saturated hydraulic conductivity $\mathbf{k}^{\mathbf{q s}}$ is a function of the saturated hydraulic conductivity $\mathbf{k}^{\text {sat }}$, the minimum quasi-saturated hydraulic conductivity $\mathbf{k}_{\mathbf{0}}$, which is the hydraulic conductivity at the transition from a quasi-saturated to an unsaturated state, i.e. at suction values slightly smaller than the air entry value, the volumetric content of entrapped air $\omega$ and the maximum entrapped air content $\omega_{\max } . \omega_{\max }$ is the volumetric content of entrapped air, which corresponds to the minimum quasi-saturated hydraulic conductivity $\mathbf{k}_{\mathbf{0}}$. $n$ is a fitting parameter [4]. The relative hydraulic conductivity $k_{\text {rel }}$ in Equation (1) is the ratio between the quasi-saturated hydraulic conductivity and the saturated hydraulic conductivity.
A comparison of different laboratory tests on the hydraulic conductivity of quasi-saturated soils [4] showed a significant difference in the relative hydraulic conductivity between coarse soils, like sand, and finegrained soils - see Figure 2. The entrapped air saturation is the volumetric air content divided by the porosity.

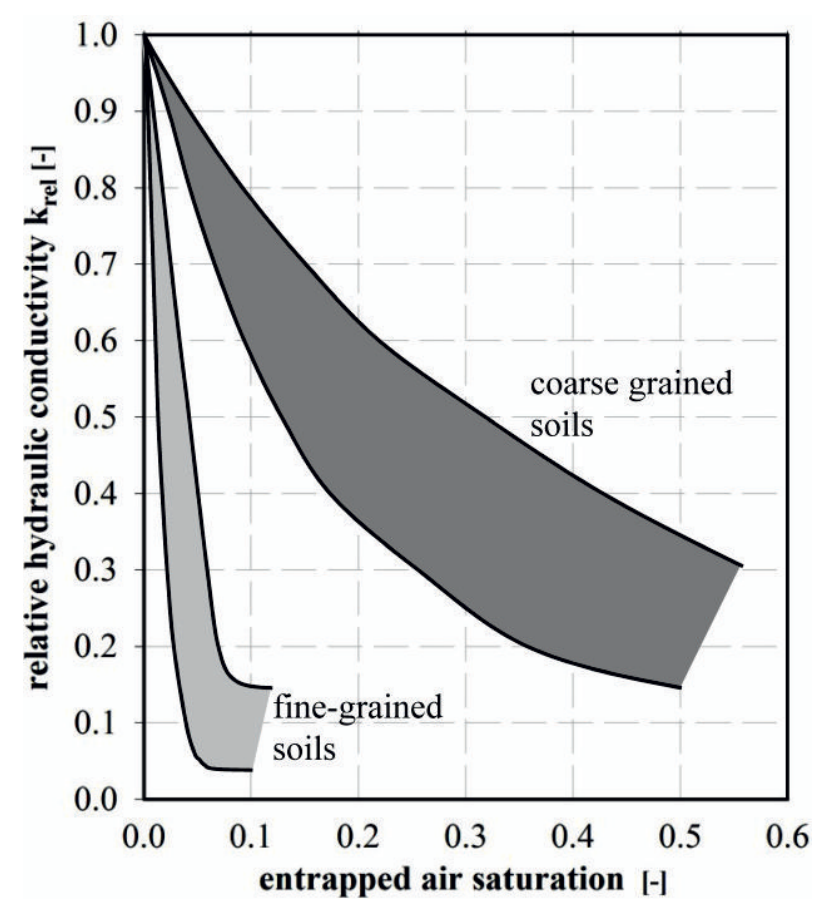

Figure 2. Relative hydraulic conductivity of quasi-saturated soils. Adapted according to [4].

\subsection{Water retention curve of quasi-saturated soils}

Water retention curves for quasi-saturated soils can be derived either from laboratory tests or on the basis of theoretical approaches. Recent literature provides different formulations of the WRC [6, 3, 2].

The WRC for a soil with positive pore water pressure $p_{w}$ and entrapped air bubbles can be derived on the basis of Boyle's law and Henry's law for dissolving of free air into water.

$$
S\left(p_{w}\right)=\frac{1}{1-h+\frac{P_{a}}{p_{w}+P_{a}} \cdot \frac{S_{\left(p_{w}=0\right)} \cdot(h-1)+1}{S_{\left(p_{w}=0\right)}}}
$$

The degree of saturation $S$ depends on Henry's parameter $h$, the atmospheric pressure $P_{a}$, the pore water pressure $p_{w}$ and a reference degree of saturation $S_{(p w=0)}$ at a pore water pressure $p_{w}=0$. Equation (3) is equal to the WRC, which was derived by Boutonnier [2]. For the derivation of the WRC a pore air pressure $p_{a}$ in the air bubbles equal to the surrounding pore water pressure $p_{w}$ was assumed. That means the influence of the surface tension is neglected. Furthermore, the vapour pressure in the air bubble is neglected. 


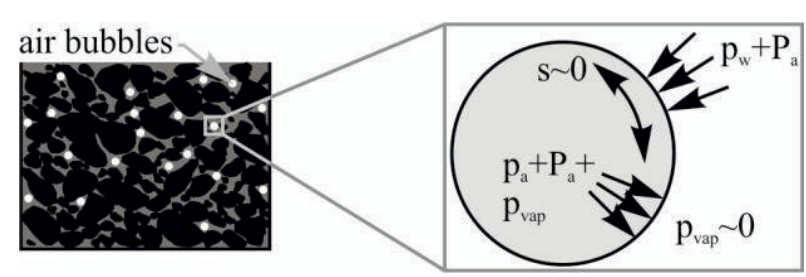

Figure 3. Assumptions for pressure in air bubble: surface tension $\mathrm{s} \sim 0$ and vapour pressure $\mathrm{p}_{\text {vap }} \sim 0$.

Henry's parameter for dissolving of free air in water can be assumed to $h=0.2$. For fast loadings, this value should be set to zero, as the dissolving of free air in water needs some time under sustained pressure [2].

Figure 4 shows a comparison of different WRC from the literature for an arbitrary quasi-saturated soil with $10 \%$ entrapped air at a pore water pressure $\mathrm{p}_{\mathrm{w}}=0$. It is emphasized that in this study the effect of air bubbles in the pore water is only investigated in the positive pore pressure range. A full WRC (with negative pore pressure range) is not used. Therefore Figure 4 does not represent a WRC for a particular soil type. According to Bicalho [6] the pore air pressure $p_{a}$ is different from the pore water pressure $p_{w}$. For a better comparability $\mathrm{p}_{\mathrm{a}}=\mathrm{p}_{\mathrm{w}}$ was also assumed for the WRC according to Bicalho [6] in Figure 4.

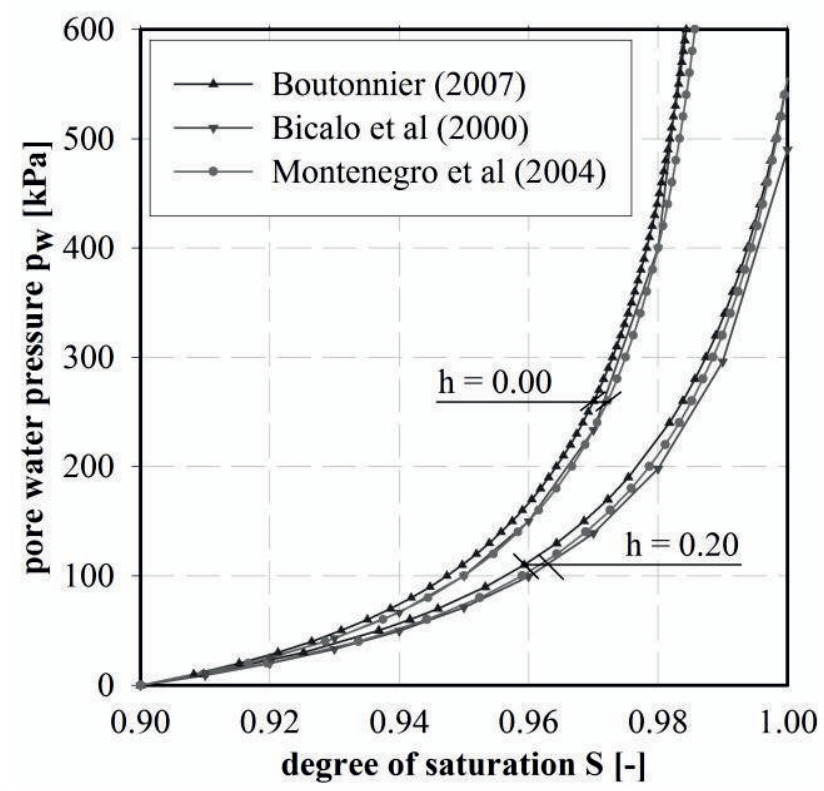

Figure 4. Comparison of water retention curves for $S_{(p w=0)}=0,9$ and $h=0.00 / 0.02$.

\subsection{Compressibility of the pore fluid}

The compressibility of the pore fluid results from the compressibility of air and the compressibility of water.

The isothermal compressibility of air $C_{a i r}$ is inversely proportional to the absolute air pressure [8]

$$
C_{a i r}=\frac{1}{P_{a}+p_{a}}
$$

$P_{a}$ is the atmospheric pressure and $p_{a}$ the pore air pressure.
The compressibility of pure water $C_{\text {water }}$ is about $4.5 \times 10^{-7}[1 / \mathrm{kPa}]$.

The compressibility of the pore fluid, i.e. of the airwater mixture, can be derived based on the proportions of the constituents air and water [8].

$$
\begin{gathered}
C_{\text {fluid }}\left(p_{w}\right)=S\left(p_{w}\right) \cdot C_{\text {water }}+\left(1-S\left(p_{w}\right)+h \cdot S\left(p_{w}\right)\right) \cdot C_{\text {air }} \\
C_{\text {fluid }}\left(p_{w}\right)=S\left(p_{w}\right) \cdot 4.5 \cdot 10^{-7}+\cdots \\
\ldots .\left(1-S\left(p_{w}\right)+h \cdot S\left(p_{w}\right)\right) \cdot \frac{1}{P_{a}+p_{w}}
\end{gathered}
$$

As the degree of saturation and the compressibility of air $C_{\text {air }}$ are a function of the pore water pressure $p_{w}$, also the compressibility of the pore fluid $C_{\text {fluid }}$ is a function of the pore water pressure $p_{w}$. In case of $\mathrm{h}=0.0$, Equation (5) is consistent with the determination of the pore fluid compressibility according to Koning and Verruijt [8].

\section{Comparison of finite element analysis and pore pressure measurements}

Deformation measurements (inclinometer and geodetic measurements) identified movements of a slope adjacent to a storage basin of a pumped storage power plant. In order to identify the causes of the slope movements, additional pore pressure gauges were installed at the toe of the slope.

The results of the pore pressure measurements could not be explained on the basis of conventional soil mechanics. Thus, in a first finite element analysis with the software PLAXIS 2D 2015 [5] the pore fluid was no longer assumed to be incompressible. A simple 1D-finite element analysis was performed to check whether the compressibility of the pore fluid, due to air bubbles in the pore water, may be the reason for the at first unexpected measurement results. Of course the 1D-model cannot capture the influence of slope movements on the pore pressure development and its causes. However, this deficiency can be accepted at this stage of investigation as the primary objective of the finite element analysis was only a qualitative comparison with the measured trend of pore water pressure changes due to changes of water levels in the reservoir.

\subsection{Site description}

A slope with a slope angle of about $30^{\circ}$ is situated next to a storage basin. Down to a depth of $25 \mathrm{~m}$ the slope consists mainly of weathered rock. At the toe of the slope, in the storage basin, the subsurface explorations identified a $20 \mathrm{~m}$ thick layer of silty sand, which is underlain by sandy clayey silt. Figure 5 shows a crosssection through the storage basin.

The water level in the storage basin changes two to four times a day, the differences in water levels being up to $8 \mathrm{~m}$. The velocity of the water level change can be up to $25 \mathrm{~m} /$ day. 


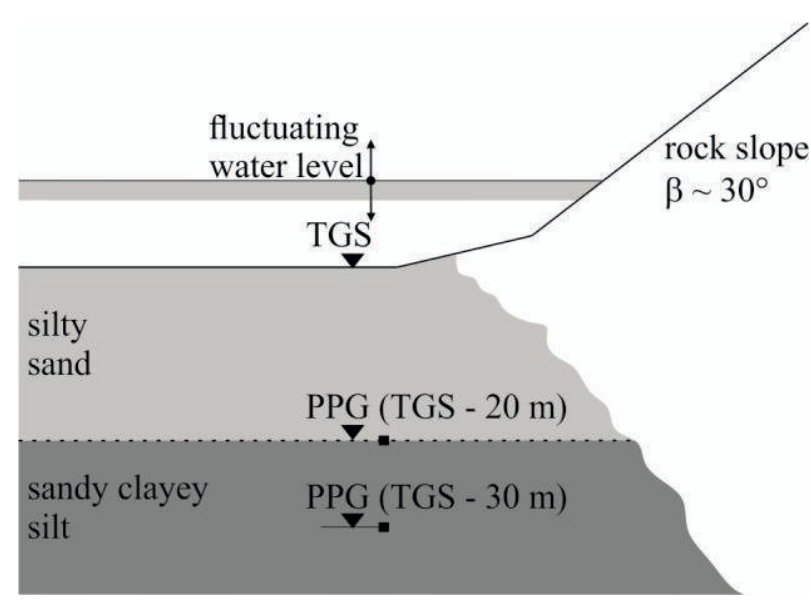

Figure 5. Cross-section through the storage basin.

\subsection{Pore pressure measurements}

The pore pressure gauges (PPG) were installed at the toe of the slope, in the storage basin, in $20 \mathrm{~m}$ and $30 \mathrm{~m}$ depth.

Figure 6 shows an example of typical pore pressure measurement results and the corresponding water level. This period was also used for the following comparative finite element analysis. The excess pore water pressure $p_{\text {excess }}$ is defined as the difference between the measured pore water pressure $p_{w}$ and the hydrostatic pore water pressure $p_{\text {hydro }}$ with reference to the general ground water table. Pressure is positive.

$$
p_{\text {excess }}=p_{\text {water }}-p_{\text {hydro }}
$$

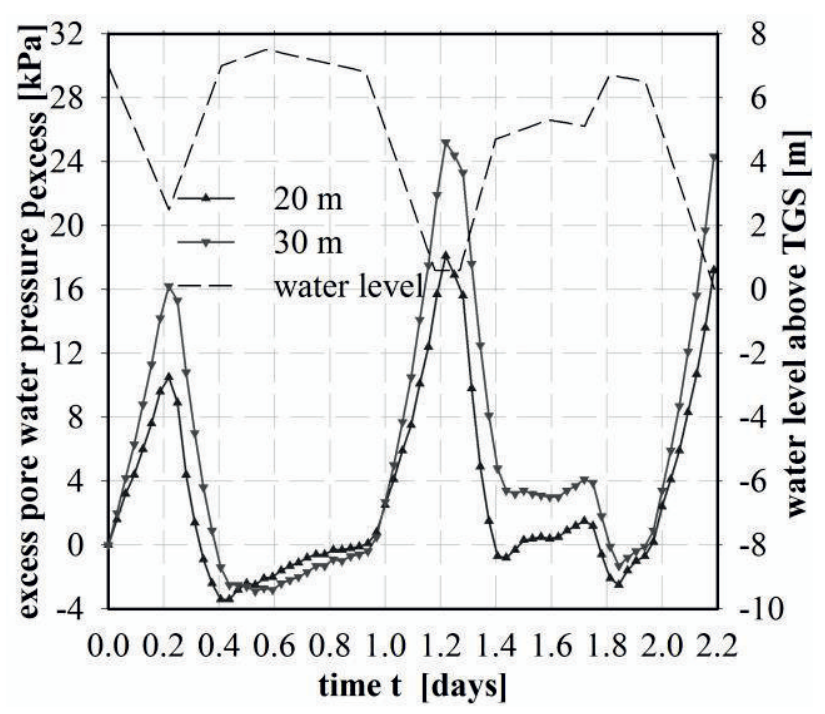

Figure 6. Typical pore pressure measurement data in $20 \mathrm{~m}$ and $30 \mathrm{~m}$ depth and corresponding water level.

In general Figure 6 shows an increasing excess pore pressure with a decreasing water level and vice versa. The value of excess pore pressure is higher the faster the water level changes. These observations might be a first indication for a compressible pore fluid as in case of quasi-saturated soils the value of the excess pore pressure is highly influenced by the ratio of loading time and hydraulic conductivity of the soil. Assuming an incompressible pore fluid, water level changes above the ground surface would not result in excess pore pressures. A further interesting detail can be observed during the period from 0.4 to 0.6 days. The recording of the water level shows a further increase in the water level but the velocity of the water level change is smaller than during the period from 0.2 to 0.4 days. These led to a further decrease of the excess pore water pressure in a depth of $30 \mathrm{~m}$. However, the measurements in $20 \mathrm{~m}$ depth show a change in the course of the excess pore water pressure. The slower increasing water level led to an increase in excess pore pressure in $20 \mathrm{~m}$ depth. This means the behaviour is different in $20 \mathrm{~m}$ and $30 \mathrm{~m}$ depth. The change of the excess pore water pressure in $20 \mathrm{~m}$ depth could be an indication for a consolidation process. The decrease in excess pore water pressure due to the following slow increasing water level is smaller than the change of the excess pore pressure towards $p_{\text {excess }}=0$ due to a reduction of the already existing excess pore pressure (consolidation). These observations led to the assumption of a compressible pore fluid for the finite element analysis.

\subsection{Finite element model}

For the qualitative comparison of the measurement results with the results of the finite element analysis a 1D-model, as shown in Figure 7, was used. The model is $30 \mathrm{~m}$ high. The upper $20 \mathrm{~m}$ consists of silty sand; the material for the lower $10 \mathrm{~m}$ is sandy clayey silt. As the permeability of the sandy clayey silt is very low the lower boundary was assumed impermeable. The boundary condition at the upper boundary is the changing water level (pore water pressure) according to the water leveltime curve in Figure 7.

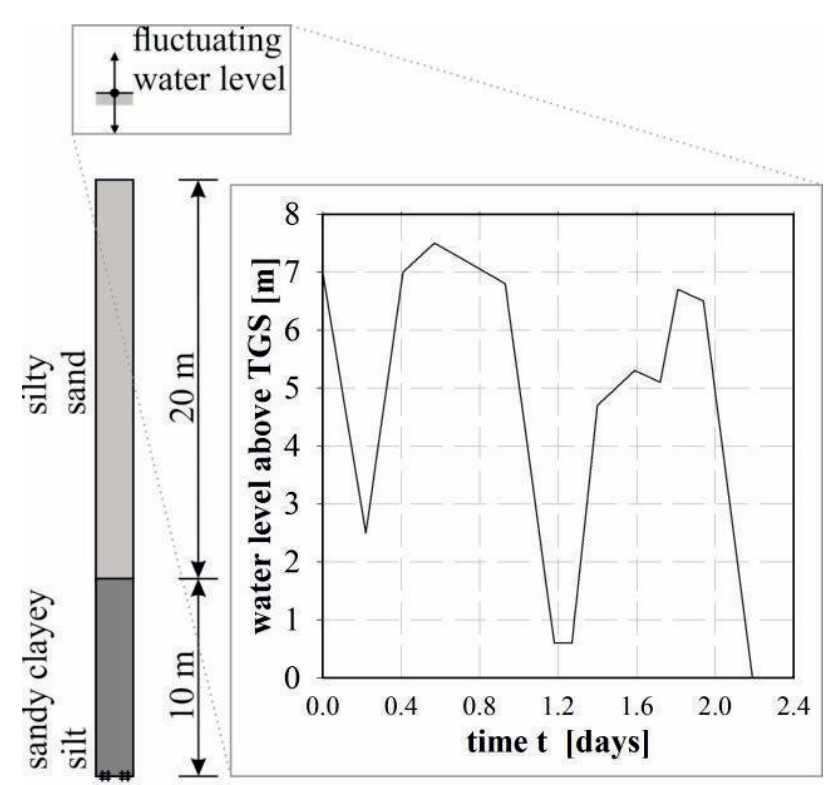
impermeable

Figure 7. 1D-finite element model and water level-time curve as upper boundary condition.

The Hardening Soil Small-model (HSS-model) [5] was used as constitutive model for both soil types. The first calculations showed that the strains, due to a 
fluctuating water level, are very small. Thus, it was necessary to use the HSS-model, which incorporates the very stiff behaviour of soils at very small strains. The soil properties were determined on the basis of laboratory tests and from experience. They are summarised in Table 1. As the qualitative pore pressure distribution is the main issue at this stage, the same mechanical properties were used for both soil types; only the assigned hydraulic conductivities are different.

Table 1. Soil properties for the Hardening Soil Small-model.

\begin{tabular}{|c|c|c|c|}
\hline Parameter & Unit & Silty sand & $\begin{array}{c}\text { Sandy clayey } \\
\text { silt }\end{array}$ \\
\hline$\gamma_{\text {unsat }}$ & $\mathrm{kN} / \mathrm{m}^{3}$ & \multicolumn{2}{|c|}{18.00} \\
\hline$\gamma_{\text {sat }}$ & $\mathrm{kN} / \mathrm{m}^{3}$ & \multicolumn{2}{|c|}{20.00} \\
\hline $\mathrm{E}_{50, \text { ref }}$ & $\mathrm{kPa}$ & \multicolumn{2}{|c|}{25000} \\
\hline$E_{\text {oed,ref }}$ & $\mathrm{kPa}$ & \multicolumn{2}{|c|}{20000} \\
\hline $\mathrm{E}_{\mathrm{ur}, \mathrm{ref}}$ & $\mathrm{kPa}$ & \multicolumn{2}{|c|}{60000} \\
\hline$v^{6}{ }_{u r}$ & - & \multicolumn{2}{|c|}{0.20} \\
\hline $\mathrm{p}_{\text {ref }}$ & $\mathrm{kPa}$ & \multicolumn{2}{|c|}{100} \\
\hline $\mathrm{m}$ & - & \multicolumn{2}{|c|}{0.80} \\
\hline$\varphi^{6}$ & $\circ$ & \multicolumn{2}{|c|}{35.00} \\
\hline$c^{6}$ & $\mathrm{kPa}$ & \multicolumn{2}{|c|}{1.00} \\
\hline$\psi^{6}$ & $\circ$ & \multicolumn{2}{|c|}{0.00} \\
\hline $\mathrm{k}^{\mathrm{sat}}$ & $\mathrm{m} / \mathrm{sec}$ & $10^{-6}$ & $10^{-8}$ \\
\hline $\mathrm{G}_{0} / \gamma_{0.7}$ & $\mathrm{kPa} /-$ & \multicolumn{2}{|c|}{$62500 / 20^{-4}$} \\
\hline
\end{tabular}

\subsection{Relative hydraulic conductivity and water retention curve}

As the velocity of the water level changes were relatively fast, Henry's parameter was assumed to be zero. Thus, the water retention curve only depends on the reference degree of saturation $S_{(p w=0)}$. The calibration of this parameter was done on the basis of the measurement data from the first lowering in water level. Compared to the measurement data, a reference degree of saturation $\mathrm{S}_{(\mathrm{pw}=0)}=0.987$ led to similar excess pore water pressures in the finite element analysis for the first water level lowering.

A parameter study showed that the influence of the relative hydraulic conductivity on the results is not very significant. This is due to the very small amount of air bubbles in the pore water $\left(\mathrm{S}_{(\mathrm{pw}=0)}=0.987\right)$. Furthermore, no data for the relative hydraulic conductivity was available. Thus relative hydraulic conductivity $k_{r e l}=1.00$ was assumed.

\subsection{Results and comparison}

Figure 8 shows a comparison of the measurement data with the results from the finite element analysis. For the comparison, the calculated change in the pore water pressure with time in $20 \mathrm{~m}$ and $30 \mathrm{~m}$ depth were analysed. Based on the calculated pore water pressure the excess pore water pressure was determined according to Equation (6).

The results of the finite element analysis are in a good agreement with the measurement data. For the period from 1.4 to 2.0 days the finite element analysis results show a slightly larger mismatch of analysis and measurements at a depth of $20 \mathrm{~m}$. The reason for this could not be identified as yet. However, it has to be kept in mind that the measurements are real field data and thus some uncertainties in the data exist, e.g. due to inhomogeneity in the subsoil. Furthermore, one of the main sliding surfaces was detected in the depth of $20 \mathrm{~m}$, thus this difference could be due to the movements of the adjacent slope, which cannot be considered within the 1D-model.

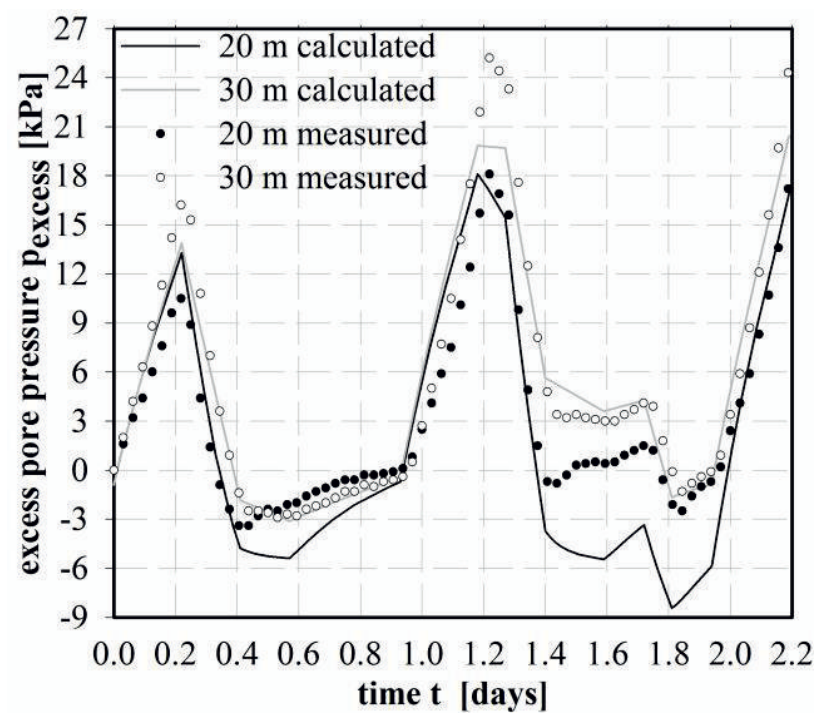

Figure 8. Comparison of measurement data with results from the finite element analysis.

In addition to the amount of air bubbles, the value of the excess pore water pressure depends mainly on the ratio between the velocity of the water level change and the hydraulic conductivity of the soil.

The change of the excess pore water pressures in a depth of $20 \mathrm{~m}$ during the period from 0.4 to 0.6 days, which is shown in the measurement data, cannot be modelled with the finite element model in very detail. However, the calculation results show a slower decrease of the excess pore water pressure in $20 \mathrm{~m}$ depth, compared to the calculation results in $30 \mathrm{~m}$ depth. This indicates a consolidation process during the further increase of the water level with a slower velocity as 
described before. The same behaviour can be observed during the period from 1.4 to 1.6 days.

\section{Conclusion and Outlook}

The presented example show that the presence of air bubbles in the pore water can have a significant influence on the development of excess pore pressures in soil layers below a reservoir with fluctuating water levels. The pore pressure measurements can be explained in a qualitative manner if the compressibility of the pore fluid, due to a small amount of air bubbles in the pore water, is considered in the finite element analysis. Eventually the excess pore water pressures could lead to a failure in the subsoil and therefore a realistic prediction of excess pore water pressures is essential in order to assess the risk of slope failure.

Further finite element analyses are currently performed including the real geometry of the slope in the vicinity of the storage basin. Finally, analyses considering full coupling of hydraulic and mechanical behaviour of the soil will be performed in order to obtain a reliable estimate of slope movements and possible failure scenarios.

\section{References}

1. B.A. Faybishenko, Water Resour. Res 31, 2421 (1995)

2. L. Boutonnier, $\mathrm{PhD}$ Thesis (Institut National Polytechnique de Grenoble, 2007)

3. H. Montenegro, H.-J. Köhler, T. Holfelder, Unsaturated Soils: Numerical and Theoretical Approaches Vol. 2 (Springer, Berlin, 2003)

4. M. Marinas, J. W. Roy, J. E. Smith, Ground Water 51/1, 41 (2013)

5. R.B.J. Brinkgreve, S. Kumarswamy, W.M. Swolfs, Manual Plaxis 2D 2015 (Plaxis bv, Delft, 2015)

6. K. V. Bicalho, D. Znidarcic, H.-Y. Ko, Geotech. SP. 99, 517 (2000)

7. A. Sakaguchi, T. Nishimura, M. Kato, Vadose. Zone J. 4, 139 (2005)

8. D. G. Fredlund, H. Rahardjo, Soil Mechanics for Unsaturated Soils (John Wiley \& Sons, New York, 1993) 